SHORT REPORT

\title{
Blink reflex in primary lateral sclerosis
}

\section{G Podikoglou, T G Avramidis, A L Papadimitriou}

J Neurol Neurosurg Psychiatry 2004;75:1064-1065. doi: 10.1136/jnnp.2003.017954

\begin{abstract}
Objective: To investigate the underlying pathophysiology of primary lateral sclerosis (PLS) regarding possible changes in blink reflex (BR) circuit.

Methods: BR was studied in 10 patients in whom diagnosis of PLS was established by comparing them to two control groups: ten patients with amyotrophic lateral sclerosis (ALS) and 30 healthy volunteers. All patients were followed up annually for a 5 year period. A typical evoked potential study of the BR was employed.

Results: Significantly lower values of $R_{2}$ and $R_{2}^{\prime}$ amplitude were found both in the PLS and ALS groups compared with the healthy control group. All these differences were highly significant $(p<0.001) . R_{1}$ amplitude and latency, as well as $R_{2}$ and $R_{2}$ ' latencies, were normal in all patients. Annually recorded follow up data revealed no significant changes in $B R$ values for PLS patients. In contrast, for ALS patients, BR progressively disappeared following clinical impairment. Conclusions: The study suggests that BR is abnormal in PLS. It also adds some clues regarding PLS pathophysiology and can be helpful as a marker of ALS progression.
\end{abstract}

$\mathrm{S}$ ince the first descriptions in the 19th century, ${ }^{12}$ many efforts have been made to support the existence of primary lateral sclerosis (PLS) as a distinct clinical entity. ${ }^{3-5}$ Early clinical diagnosis, important for prognostic considerations, has been always guarded because it is a diagnosis of exclusion proven only at autopsy. In recent years, however, modern diagnostic techniques and pathological verification made the clinical diagnosis of PLS more permissible. Moreover, new diagnostic criteria have been suggested ${ }^{6}$ in order to replace those put forward prior to the availability of modern diagnostic techniques. ${ }^{7}$

Blink reflex (BR) is a sensitive neurophysiological method to detect lesions in the pons and lateral medulla or lesions elsewhere indirectly influencing the excitability of the polysynaptic connection. ${ }^{8}$

The purpose of the present study was to examine, for the first time, BR in PLS patients. All data were compared with those of amyotrophic lateral sclerosis (ALS) patients and healthy controls.

\section{PATIENTS AND METHODS}

Ten patients (four men and six women, aged 46-66 years) with PLS were recruited after the diagnosis was first established, following nursing, at the Neurological clinic of Red Cross Hospital, Athens, Greece.

Median age of onset was 56 years. All patients underwent a 5 year follow up and extensive laboratory, radiological, and neurophysiological investigation in order to rule out known specific causes for pyramidal tract involvement. All gave oral informed consent to participation in the protocol approved by the local ethics committee. All of them fulfilled the clinical and laboratory criteria for PLS proposed by Pringle et al $1992 .{ }^{6}$
We used 10 patients with mixed form (bulbar and spinal involvement) of ALS and 30 healthy volunteers as controls matched for age and sex.

All patients declared that they had developed symptoms for a maximum of 6 months. Neither patients nor the healthy controls received any medication prior to BR testing.

A typical evoked potential study of the BR was employed (intensity stimulus $10-12 \mathrm{~mA}$, square wave pulse duration $0.2 \mathrm{msec}, \mathrm{BPF} 20 \mathrm{~Hz}-3 \mathrm{kHz}$ ).

Both patients and controls were stimulated on the right side of the head and recorded on both sides. Eight recordings were made for each side-the time interval between successive trials being more than 30 seconds to reduce the possibility of habituation. Finally, mean values were calculated for each side. We compared latency ( $\mathrm{msec}$ ) and peak-topeak amplitude $(\mu \mathrm{V})$ of $\mathrm{R}_{1}, \mathrm{R}_{2}$, and $\mathrm{R}_{2}{ }^{\prime}$ between patient groups and healthy controls, and between PLS and ALS patients.

Comparison between controls, PLS, and ALS patients was made using Kruskal-Wallis test. Comparison between specific groups was made using Mann-Whitney U test. Follow up data were analysed with linear regression analysis.

\section{RESULTS}

All PLS and ALS patients had statistically significantly lower amplitude of ipsilateral $\mathrm{R}_{2}$ and contralateral $\mathrm{R}_{2}{ }^{\prime}$ when compared with controls $(\mathrm{p}<0.001)$. In contrast, there were no statistically significant differences in BR responses $(\mathrm{p}>0.1)$ with PLS compared with ALS patients.

$R_{1}, R_{2}$, and $R_{2}{ }^{\prime}$ latencies, as well as $R_{1}$ amplitude, were normal in all patients. Table 1 demonstrates the values of $R_{1}$, $\mathrm{R}_{2}$, and $\mathrm{R}_{2}{ }^{\prime}$ components in the PLS group versus the ALS and the healthy control groups. As already mentioned, all PLS patients were re-tested at least every year during the 5 year follow up. For this period, however, clinical follow up findings and BR data did not significantly worsen.

Conversely, electrophysiological follow up data from ALS patients revealed that BR abnormalities did progress with time. With the quick process of the disease (based on our clinical follow up findings), $R_{2}$ and $R_{2}{ }^{\prime}$ latencies were prolonged, and finally BR components disappeared, beginning with $R_{2}{ }^{\prime}$ followed by $R_{2}$ and $R_{1}$. At the late stage of the disease (locked-in state) we were not able to record BR.

\section{DISCUSSION}

Adults with slowly progressive non-inherited gait disorders may show no abnormalities on examination other than signs implicating the corticospinal tract. That is the syndrome of PLS, a clinical diagnosis that has been guarded because it is a diagnosis of exclusion proven only at autopsy. The clinical features are limited to those associated with dysfunction of the descending motor tracts and include spastic quadriparesis, pseudobulbar affect, spastic dysarthria, hyper-reflexia,

Abbreviations: ALS, amyotrophic lateral sclerosis; BR, blink reflex; PLS, primary lateral sclerosis 
Table 1 Latency and amplitude of $R_{1}, R_{2}$, and $R_{2}$ ' components of BR in the PLS group $(n=10) \vee A L S$ group $(n=10)$ and controls $(n=30)$. Values are mean (SD).

\begin{tabular}{llll}
\hline & & \multicolumn{3}{l}{ Patients } \\
\cline { 3 - 4 } Variable & Controls & PLS & ALS \\
\hline lpsilateral responses & & & \\
$\mathrm{R}_{1}$ latency $(\mathrm{msec})$ & $10.6(1.1)$ & $10.2(0.5)$ & $10.1(0.5)$ \\
$\mathrm{R}_{1}$ amplitude $(\mu \mathrm{V})$ & $250(48.2)$ & $232.4(41.8)$ & $231.5(46.4)$ \\
$\mathrm{R}_{2}$ latency $(\mathrm{msec})$ & $31.1(2.2)$ & $30.8(1.6)$ & $30.6(1.5)$ \\
$\mathrm{R}_{2}$ amplitude $(\mu \mathrm{V})$ & $345(81.2)$ & $158.6(50.3)^{*}$ & $151.2(48.8)^{*}$ \\
Contralateral responses & & & \\
$\mathrm{R}_{2}{ }^{\prime}$ latency $(\mathrm{msec})$ & $31.3(2.3)$ & $31.25(1.6)$ & $30.9(1.5)$ \\
$\mathrm{R}_{2}{ }^{\prime}$ amplitude $(\mu \mathrm{V})$ & $296.2(62.3)$ & $92.7(22.2)^{*}$ & $91(21.6)^{*}$ \\
\hline
\end{tabular}

${ }^{*} \mathrm{p}<0.001 v$ healthy control group and $\mathrm{p}>0.1 \mathrm{v}$ other patient group. AML, amyotrophic lateral sclerosis; PLS, primary lateral sclerosis.

and bilateral Babinski signs. Muscular atrophy and fasciculations are uniformly absent. ${ }^{6}$

It is well known that changes of $\mathrm{R}_{2}$ component may imply lesions directly affecting the reflex pathways per se, as in the case of Wallenberg syndrome, or lesions elsewhere indirectly influencing the excitability of the polysynaptic connections. ${ }^{8}$

BR studies may show absent or markedly diminished $\mathrm{R}_{2}$ and $\mathrm{R}_{2}{ }^{\prime}$ components with normal or nearly normal $\mathrm{R}_{1}$ in patients with hemispheric lesions, pseudobulbar palsy, etc. ${ }^{9}$

$\mathrm{BR}$ responses are mediated through pons and lateral medulla and the possibility to be compromised by the PLS degenerative process is high. Based on this hypothesis, we studied, for the first time, BR in a group of patients in which the diagnosis of PLS was permissible as they fulfilled the clinical and laboratory criteria suggested by Pringle et al. ${ }^{6}$

Our results demonstrate that BR is abnormal (significantly lower values of $\mathrm{R}_{2}$ and $\mathrm{R}_{2}{ }^{\prime}$ amplitude) in the PLS and ALS groups compared with healthy controls. Few papers concerning BR in ALS have been published. ${ }^{10}$ Lower amplitudes and prolonged latencies of $\mathrm{R}_{2}$ and $\mathrm{R}_{2}{ }^{\prime}$ have been reported. We confirmed these results in our paper, except that prolonged latencies were observed only in a later stage. Recently it was shown that the $\mathrm{R}_{2}$ component consists of distinct subcomponents. ${ }^{11}$ We were not able, however, to separate distinct $R_{2}$ subcomponents either from patients or from controls. This can be explained from their temporal overlap provided that our recordings were conventional.

$\mathrm{R}_{1}$ amplitude was normal in all cases. This dissociation between $R_{1}$ and $R_{2}$ amplitude indicates an excitability change of interneurones rather than motorneurones, because $R_{1}$ is oligosynaptic whereas $R_{2}$ is polysynaptic. Regarding pathophysiology, the abnormal values of $R_{2}$ and $R_{2}{ }^{\prime}$ amplitudes is indeed interesting because the $R_{2}$ suppression in suprasegmental lesions is often attributed to a diffuse depression of the corticoreticular drive. The present findings indicate that pyramidal projections from the precentral gyrus to the medullary lateral reticular formation exert a strong facilitatory influence.

As $R_{1}, R_{2}$, and $R_{2}{ }^{\prime}$ latencies are within normal limits, we must conclude that the BR arc is anatomically intact. If the suprasegmental lesion, however, acts by reducing the excitability of the interneurones in the circuit, we will expect an increase in the synaptic time. Indeed, in stroke hemiplegia the $\mathrm{R}_{2}$ latency is increased.

In PLS, however, the dissociation between bilateral $R_{2}$ amplitude and latency, and the fact that BR abnormalities did not progress with time during the whole 5 year follow up, can be perhaps attributed to the slow progression of the disease itself.

In contrast, for ALS patients, the prolonged $R_{2}$ and $R_{2}^{\prime}$ latencies (only present at a later stage of the disease), and the progressive disappearance of BR components, indicate a fast degeneration process that can be possibly attributed to additional involvement of the lower motor neurone and not only reflect progressive degeneration of the central nervous system.

Because there were no statistically significant differences in BR components between first diagnosed PLS and ALS patients, both being abnormal, we cannot use BR as an additional diagnostic criterion suggestive of PLS.

We can only propose that following up BR provides us with valuable clues concerning PLS pathophysiology. It can also be helpful as a marker of ALS progression.

The limited number of cases, and the rarity of the disease itself, raises the need for our results to be verified with larger number of cases and to be followed up for a longer period of time.

\section{Authors' affiliations}

D G Podikoglou, T G Avramidis, Department of Neurology, Red Cross Hospital, Athens, Greece

A L Papadimitriou, Department of Neurology, University of Thessaly, Thessaly, Greece

Correspondence to: Danis G Podikoglou, 68 Spetson Street, Kipseli, Athens 11362, Greece; danisgr@teledomenet.gr

Received 1 May 2003

In revised form 15 November 2003

Accepted 17 November 2003

\section{REFERENCES}

1 Charcot JM. Sclerose des cordons lateraux de la moelle epiniere chez une femme hysterique, atteinte de contracture permanente des quatre membres. Bull Mem Soc Med Hop Paris [Second series] 1865;2:24-42.

2 Erb WH. Uber einen wenig bekannten spinalen Symptomencomplex. Berliner Klinische Wochenschriff 1875;12:257-359.

3 Fisher CM. Pure spastic paralysis of corticospinal origin. Can J Neurol Sci 1977;4:251-8.

4 Beal MF, Richardson EP. Primary lateral sclerosis: a case report. Arch Neurol $1981 ; 38: 630-3$.

5 Younger DS, Chou S, Hays AP, et al. Primary lateral sclerosis. A clinical diagnosis reemerges. Arch Neurol 1988;45(12):1304-7.

6 Pringle CE, Hudson AJ, Munoz DG, et al. Primary lateral sclerosis. Clinical features, neuropathology and diagnostic criteria. Brain 1992;115(Pt 2): 495-520.

7 Stark FM, Moersch FP. Primary lateral sclerosis: a distinct clinical entity. J Nerv Ment Dis 1945;102:332-37.

8 Kimura J, Lyon LW. Orbicularis oculi reflex in the Wallenberg syndrome: alterations of the late reflex of the spinal tract and nucleus of the trigeminal nerve. J Neurol Neurosurg Psychiatry 1972;35:228-33.

9 Kimura J. Electrodiagnosis in diseases of nerve and muscle: principles and practice. Philadelphia: F A Davis Company, 1983.

10 Shimoda M, Yokohama Y, Okada A, et al. Electrically induced blink reflex and clinical blinking ability in patients with amyotrophic lateral sclerosis. Acta Neurol Scan 1995;92:141-4.

11 Meincke U, Topper R, Gouzoulis-Mayfrank E. The electrically elicited R $R_{2}$ blink reflex consists of distinct subcomponents. Electromyogr Clin Neurophysiol 2003;43(2):91-5 